\title{
A MODEL OF OPTICAL BISTABILITY AND POLARISATION EVOLUTION IN BIREFRINGENT $\chi^{(2)}$ NONLINEAR MEDIA
}

\author{
W. JEูDA ${ }^{a}$ AND A. ŻAGóRSKI ${ }^{b}$ \\ a Systems Research Institute, Polish Academy of Sciences \\ Newelska 6, 01-447 Warszawa, Poland \\ ${ }^{6}$ Institute of Physics, Warsaw University of Technology \\ Koszykowa 75, 00-662 Warszawa, Poland
}

(Received April 14, 1999; in final form June 9, 1999)

\begin{abstract}
We present a model calculation of optical bistability and polarisation evolution in optically birefringent media based on the interaction of three waves. Second-order optical nonlinearity (described by the third rank susceptibility tensor) is assumed to be dominant. A bistable light transmission is analysed in terms of standard Fabry-Perot model of feedback, and is a direct consequence of the results obtained for a semi-infinite medium. A second effect considered in the paper is evolution of polarisation state of the waves involved in these processes. We derived a closed formula for intensity-dependent refractive index, which corresponds to that used in the case of third-order nonlinear media. As a result, we calculated both intensity and Stokes parameters of transmitted light.
\end{abstract}

PACS numbers: 42.65.Pc, 42.65. Hw

\section{Introduction}

Optical bistability was first discovered in media with the third-order nonlinearity. With such a nonlinearity the refractive index depends on the light intensity. The physical background of this phenomenon is described in many textbooks (see [1-3] for a review). On the other hand, the second-order bistability has focused much less attention [4-8]. The mechanisms leading to it are different from those of the third-order bistability. To explain second-order bistability it seems necessary to consider the so-called cascade-process $[9,10]$, which is connected with the second-harmonic generation. The generated wave of frequency $2 \omega$ is of a weak intensity and does not essentially change the amplitude of the incoming wave of frequency $\omega$. However, its influence on the phase of the incoming wave is crucial. This phase change, together with an appropriate feedback and non-vanishing phase mismatch, is sufficient for bringing about the intensity bistability. 
In a more general case, when the sample is illuminated by two waves of frequencies $\omega_{1}$ and $\omega_{2}$, with $\omega_{1}>\omega_{2}$, the role of the second-harmonic wave is taken over by the wave with frequency $\omega_{3}=\omega_{1}-\omega_{2}$. This wave changes the phases of the two incoming waves in such a way that their intensities exhibit bistability.

The intensity-dependent phase change results also in evolution of the polarisation state. This may lead to a completely different behaviour as compared to the linear case, including polarisation constancy (along the propagation direction) and polarisation bistability (with regard to the input intensity).

\section{Degenerated three waves interaction in second-harmonic generation regime}

This paper deals with birefringent crystals for which the condition of the phase-matching may not be fully satisfied (e.g. $n^{(0)}(\omega)<n^{(e)}(2 \omega)$ for negative birefringence). In such a case the process of the second-harmonic generation can be maximised by appropriate orientation of the sample. It corresponds to the propagation of the fundamental wave in the clirection (the $O z$ axis) which is perpendicular to the main optical axis (assumed further as the $O y$ axis).

We assume that the sample of nonlinear crystal is illuminated by a strong laser beam normally to the surface of the film. The wave is characterised by the frequency $\omega$, the wave vector $k_{\omega}=\left(0,0, k_{\omega}\right)$ parallel to the $z$-axis of Cartesian coordinate system, and the electric vector $\boldsymbol{E}_{\omega}=\left(E_{\omega}^{x}, E_{\omega}^{y}, 0\right)$. Inside the crystal the wave vector maintains its direction and remains perpendicular to $\boldsymbol{E}$.

The condition of the smallest phase mismatch in the process of second-harmonic generation in (negatively) birefringent media leads to the following processes:

$$
E_{\omega}^{(\mathrm{o})}+E_{\omega}^{(\mathrm{o})} \rightarrow E_{2 \omega}^{(\mathrm{e})}, \quad E_{\omega}^{(\mathrm{o})}+E_{2 \omega^{\prime}}^{(\mathrm{e})}-E_{\omega}^{(\mathrm{o})} .
$$

The ordinary wave is always polarised in the direction which is normal to the plane defined by the main axis (here $O y$ axis) and $k$ vector, whereas the extraordinary wave lies in that plane. Within this convention we may write

$$
\begin{aligned}
& E_{\omega}^{x}=E_{\omega}^{(\circ)}, \quad E_{\omega}^{y}=E_{\omega}^{(e)}, \\
& E_{2 \omega}^{x} \equiv 0, \quad E_{2 \omega}^{y}=E_{2 \omega}^{(\mathrm{e})} .
\end{aligned}
$$

The component $E_{\omega}^{(e)}$ of the fundamental wave is not involved in the nonlinear interaction considered, hence its evolution follows the rules of linear optics.

The second-order nonlinearity of the material is described by the third-order susceptibility tensor $\chi^{(2)}$, which appears in the relation: $\boldsymbol{P}^{\mathrm{NL}}=\chi^{(2)} \boldsymbol{E} \boldsymbol{E}$. According to (1) only two components of $\chi^{(2)}$ are involved: $\chi_{x x y}^{(2)}$ and $\chi_{y x x}^{(2)}$. These may, however, depend on the frequency. In the absence of absorption, Kleinman symmetry relations apply and then we can express both components by one parameter $\gamma$ as follows:

$$
\frac{1}{2} \chi_{x x y}^{(2)}(\omega)=\chi_{y x x}^{(2)}(2 \omega) \equiv \gamma
$$


Due to the nonlinearity the two waves $\boldsymbol{E}_{1}$ and $\boldsymbol{E}_{2}$ of frequencies $\omega$ and $2 \omega$, respectively, are coupled and there appears a mutual energy transfer. This energy transfer may exhibit periodicity along the $z$-axis and was recently named the cascaded process. It was suggested in [11-13] that the cascaded process may lead to nonlinear phase shifts of the fundamental frequency and of the second-harmonic beam. Since dispersive optical bistability is usually described in terms of the phase shift arising from the Kerr nonlinearity, a bistable behaviour based on nonlinear phase shift in the cascading process is also expected.

In the slowly-varying-envelope approximation, Maxwell's equations applied to a noncentrosymmetric and non-absorbing crystal, lead to two coupled amplitude equations, which govern the second-harmonic generation process

$$
\begin{aligned}
& \frac{\mathrm{d} E_{1}}{\mathrm{~d} z}=-\mathrm{i} \kappa_{1} E_{2} E_{1}^{*} \exp (\mathrm{i} \Delta k z), \\
& \frac{\mathrm{d} E_{2}}{\mathrm{~d} z}=-\mathrm{i} \kappa_{2} E_{1}^{2} \exp (-\mathrm{i} \Delta k z),
\end{aligned}
$$

where $E_{1}$ and $E_{2}$ are complex electric fields of the incoming (fundamental) and the second-harmonic beam, respectively (the asterisk denotes the conjugate complex quantity),

$$
\Delta k=k_{2}-2 k_{1}=\frac{2 \omega}{c}\left(n_{2}^{\mathrm{L}}-n_{1}^{\mathrm{L}}\right)
$$

is the wave-vector mismatch and

$$
\kappa_{j}=\frac{\omega \mu_{0} c}{2 n_{j}} \gamma \quad(j=1,2),
$$

where $\mu_{0}$ denotes the permeability of vacuum, $n_{j}^{\mathrm{L}}$ are the (linear) refraction indices of the two waves of frequencies $\omega$ and $2 \omega, c$ is the speed of light in vacuum, and $\gamma$ is defined in (3). Since $n_{2}-n_{1}>0$, the wave-vector mismatch is positive.

Our main assumption is that of a sufficiently large phase mismatch $\Delta k$. This implies the absence of depletion of the incident wave amplitude, but its phase $\varphi_{1}$ depends on $z$. After some algebra we obtain the following formula:

$$
\delta \varphi_{1}(I)=\frac{\kappa_{1} \kappa_{2} z}{\Delta k}\left[1-\frac{\sin (\Delta k z)}{\Delta k z}\right] I
$$

where $\delta \varphi_{1}=\varphi_{1}(z)-\varphi_{1}(0)$ and $I=\left|E_{1}\right|^{2}$.

In order to derive consequences from (7) we refer to the case of media with the third-order nonlinearity, when the refractive index $n$ is written in the form

$$
n=n^{\mathrm{L}}+n^{\mathrm{NL}} /
$$

where $n^{\mathrm{L}}$ is the linear refractive index and $n^{\mathrm{NL}}$ is a coefficient of the nonlinear part of the refractive index and $E$ denotes the electric field. By analogy, we can identify $\delta \varphi_{1}$ with $n_{1}^{\mathrm{NL}} I_{1}$, and then we get

$$
n_{1}^{\mathrm{NL}}(z)=\frac{\omega \mu_{0}^{2} c^{3} \gamma^{2}}{2 n_{1} n_{2} \Delta k}\left[1-\frac{\sin (\Delta k z)}{\Delta k z}\right] .
$$

We see that the refractive index essentially depends on the path length of the beam. Thus, the effect of the nonlinearity can be enhanced by a proper choice of the thickness of the sample. It is worthwhile to note that $n_{1}^{\mathrm{NL}}$ is always positive, as $\Delta k>0$. 


\section{Three-waves interaction in the regime of difference-wave generation}

The discussion of Sec. 2 may be extended to cover the case of interaction of three waves. We now assume that two waves of frequencies $\omega_{1}$ (pump beam) and $\omega_{2}$ (probe beam) enter a nonlinear medium. The nonlinear interaction between them leads to waves with frequencies combined from $\omega_{1}$ and $\omega_{2}$, in particular to the wave with frequency $\omega_{3}=\omega_{1}-\omega_{2}$. In the slowly-varying-envelope approximation, Maxwell's equations applied to this system lead to coupled amplitude equations

$$
\begin{aligned}
& \frac{\mathrm{d} E_{1}}{\mathrm{~d} z}=-\mathrm{i} \kappa_{1} E_{2} E_{3} \exp (\mathrm{i} \Delta k z), \\
& \frac{\mathrm{d} E_{2}}{\mathrm{~d} z}=-\mathrm{i} \kappa_{2} E_{3}^{*} E_{1} \exp (-\mathrm{i} \Delta k z), \\
& \frac{\mathrm{d} E_{3}}{\mathrm{~d} z}=-\mathrm{i} \kappa_{3} E_{2}^{*} E_{1} \exp (-\mathrm{i} \Delta k z),
\end{aligned}
$$

where $\Delta k=\left(\omega_{1} n_{1}-\omega_{2} n_{2}-\omega_{3} n_{3}\right) / c$ and $\left(\mu_{0} c \omega_{j} / n_{j}\right) \chi^{(2)}\left(\omega_{j}\right)=\kappa_{j}$. The assumption of the small pump-wave depletion $\left(I_{1}(z) \approx I_{1}=\right.$ const) requires

$$
\left[\frac{(\Delta k)^{2}}{\left(\Delta k_{\mathrm{eff}}\right)^{2}}-1\right] \frac{I_{2}(z)}{I_{1}} \ll 1
$$

where

$$
\left(\Delta k_{\mathrm{eff}}\right)^{2}=(\Delta k)^{2}-\frac{4 \mu_{0}^{2} c^{2} \gamma^{2} \omega_{2} \omega_{3}}{n_{2} n_{3}} I_{1}
$$

In what follows we are interested in two special cases: (a) $I_{2} \approx I_{1}$ and (b) $I_{2} \ll I_{1}$. In the case (a) the condition (10) implies that $\Delta k^{2} \gg\left(4 \mu_{0}^{2} c^{2} \gamma^{2} \omega_{2} \omega_{3} / n_{2} n_{3}\right) I_{1}$. It means a large wave-vector mismatch, hence the intensities of both two waves remain practically unchanged. Under this condition we can derive the following expressions for the phase shifts:

$$
\delta \varphi_{m}\left(I_{l}\right)=\frac{\mu_{0}^{2} c^{2} \gamma^{2} \omega_{m} \omega_{3} z}{n_{m} n_{3} \Delta k}\left[1-\frac{\sin (\Delta k z)}{\Delta k z}\right] I_{l} \quad(m, l=1,2 ; \quad m \neq l) .
$$

These can be related to intensity-dependent parts of refractive indices

$$
n_{m}^{\mathrm{NL}}=n_{m}^{\mathrm{NL}}(z)=\frac{\mu_{0}^{2} c^{3} \gamma^{2} \omega_{3}}{n_{m} n_{3} \Delta k}\left[1-\frac{\sin (\Delta k z)}{\Delta k z}\right], \quad m=1,2,
$$

which stand in the formulae for effective refractive indices

$$
n_{m}^{\mathrm{eff}}=n_{m}+n_{m}^{\mathrm{NL}} I_{l}
$$

We observe that $n_{1}^{\text {eff }}$ depends only on $I_{2}$, but not on $I_{2}$, and vice versa. This cross-dependence represents a new effect in comparison with the standard Kerr effect.

In the case (b) we assume that the intensity of the pump beam is much larger than that of the probe beam. The condition (10) will now be satisfied if $\Delta k^{2} \geq\left(8 \mu_{0}^{2} c^{2} \gamma^{2} \omega_{2} \omega_{3} / n_{2} n_{3}\right) I_{1}$. Following the discussion leading to (8) and (13) we obtain expressions for the intensity-dependent parts of refractive indices 


$$
n_{1}^{\mathrm{NL}}=n_{1}^{\mathrm{NL}}\left(I_{1}, z\right)=\frac{\mu_{0}^{2} c^{3} \gamma^{2} \omega_{3}}{n_{1} n_{3} \Delta k}\left(\frac{\Delta k}{\Delta k_{\mathrm{eft}}}\right)^{2}\left[1-\frac{\sin \left(\Delta k_{\mathrm{eff}} z\right)}{\Delta k_{\mathrm{eff}} z}\right]
$$

(the dependence on $I_{1}$ is hidden in $\Delta k_{\text {eff }}$; see (11)),

$$
n_{2}^{\mathrm{NL}}=n_{2}^{\mathrm{NL}}(z)=\frac{\mu_{0} c^{3} \gamma^{2} \omega_{3}}{n_{2} n_{3} \Delta k}\left[1-\frac{\sin (\Delta k z)}{\Delta k z}\right] .
$$

We observe that the formulae for intensity-dependent refractive indices $(15 \mathrm{a}, \mathrm{b})$ have a similar structure to (8) and (13). However, an important difference is that now $n_{1}^{\text {NL }}$ depends also on the corresponding pump beam intensity $I_{1}$.

\section{Optical bistability in Fabry-Perot cavity filled with $\chi^{(2)}$ medium}

The mode of reasoning in Refs. [1, 2], developed for dispersive media with third-order nonlinearity, can be extended to second-order nonlinear media provided that intensities of intracavity beams remain constant. In this case the standard expression for the transmission of the cavity is given by the well-known formula:

$$
\mathcal{I}=\frac{(1-R)^{2}}{1+R^{2}-2 R \cos \left[2 k L+\delta \varphi\left(\frac{1}{1-R} I^{\text {out }}\right)+\delta \varphi\left(\frac{R}{1-R} I \text { out }\right)\right]},
$$

where $\delta \varphi(I)$ is - for the case of SHG - given by (7).

This function has a periodic character, shown in Fig. 1. The region with triple solutions characterises the phenomenon of bistability. Increasing the parameter of nonlinearity $\gamma$ enhances the above effect, leading - in general - to multistability (Fig. 2).

When dealing with two incident waves of comparable intensities, the formula (16) is replaced by two coupled formulae of the form

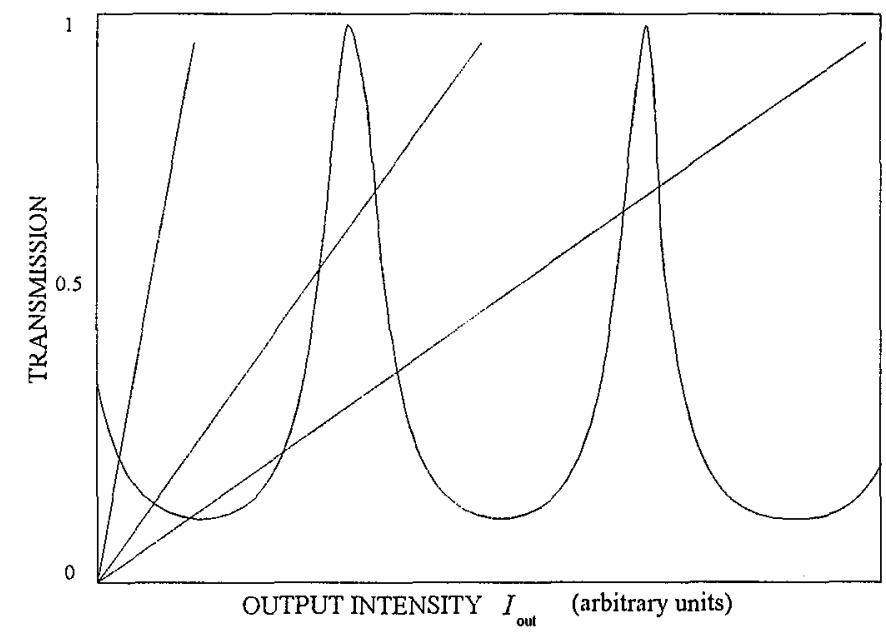

Fig. 1. Transmission of the cavity as a function of incoming beam intensity. 


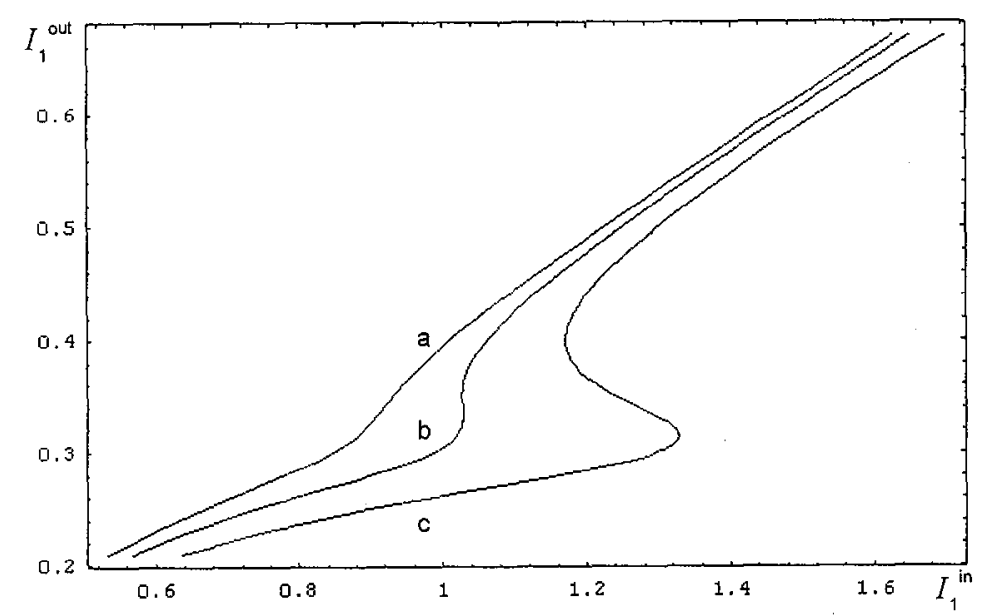

Fig. 2. Output versus input intensity of strong wave for different values of input weak-wave intensity. $a-I_{2}^{\text {in }}=0.01, b-I_{2}^{\text {in }}=0.02, c-I_{2}^{\text {in }}=0.04$ (in arbitrary units).

$$
\mathcal{I}_{m 2}=\frac{\left(1-R_{m}\right)^{2}}{1+R_{m}^{2}-2 R_{m} \cos \left[2 k_{m} L+\delta \varphi_{m}\left(\frac{1}{1-R_{l}} I_{l}^{\text {out }}\right)+\delta \varphi_{m}\left(\frac{R_{l}}{1-R_{l}} I_{l}^{\text {out }}\right)\right]}
$$

for $(m, l=1,2 ; m \neq l)$.

If $I_{2} \ll I_{1}$, it is necessary to take into account the spatial variation of the probe beam inside the cavity. This effects in the reformulation of the above expression for the pump beam (with $\mathcal{I}_{2}$ unchanged) where

$$
\mathcal{I}_{1}=\frac{\left(1-R_{1}\right)^{2} M_{\mathrm{F}}}{1+R_{1}^{2} M_{\mathrm{F}} M_{\mathrm{B}}-2 R_{1} \sqrt{M_{\mathrm{F}} M_{\mathrm{B}}} \cos \left(2 k_{1} L+\delta \varphi_{1}^{\mathrm{F}}+\delta \varphi_{1}^{\mathrm{B}}\right)},
$$

$$
\begin{aligned}
& M_{\mathrm{F}}=1+\frac{4 \kappa_{2} \kappa_{3} I_{1}^{\text {out }}}{R\left(\Delta k_{\mathrm{eff}}^{\mathrm{F}}\right)^{2}} \sin ^{2} \frac{\Delta k_{\mathrm{eff}}^{\mathrm{F}} L}{2}, \\
& M_{\mathrm{B}}=1+\frac{4 \kappa_{2} \kappa_{3} I_{1}^{\text {out }}}{\left(\Delta k_{\mathrm{eff}}^{\mathrm{B}}\right)^{2}} \sin ^{2} \frac{\Delta k_{\mathrm{eff}}^{\mathrm{B}} L}{2} .
\end{aligned}
$$

Here, $\Delta k_{\text {eff }}^{\mathrm{F}}=\Delta k_{\text {eff }}\left(\frac{1}{1-R_{1}} I_{1}^{\text {out }}\right)$ and $\Delta k_{\text {eff }}^{\mathrm{B}}=\Delta k_{\text {eff }}\left(\frac{R_{1}}{1-R_{1}} I_{1}^{\text {out }}\right)$. The phase shifts of the forward and backward waves may be calculated on the basis of the formula (15a). For the sake of simplicity we omit here the details.

In Fig. 3 we observe that changing the value of input probe beam intensity we influence the input-output characteristics of the Fabry-Perot cavity for the pump beam, from linear to bistable. We also observe (Fig. 4), that the intensity of the probe beam at the output is strongly influenced by the change of input pump beam intensity, and that the shape of this relation can exhibit bistability.

We have thus shown that the cascaded process in a Fabry-Perot cavity filled up with a material exhibiting second-order nonlinearity, in the case of sufficiently large phase mismatch, leads to the effect of intensity bistability of dispersive type. This bistability is connected with a length-of-path dependent refractive index. 


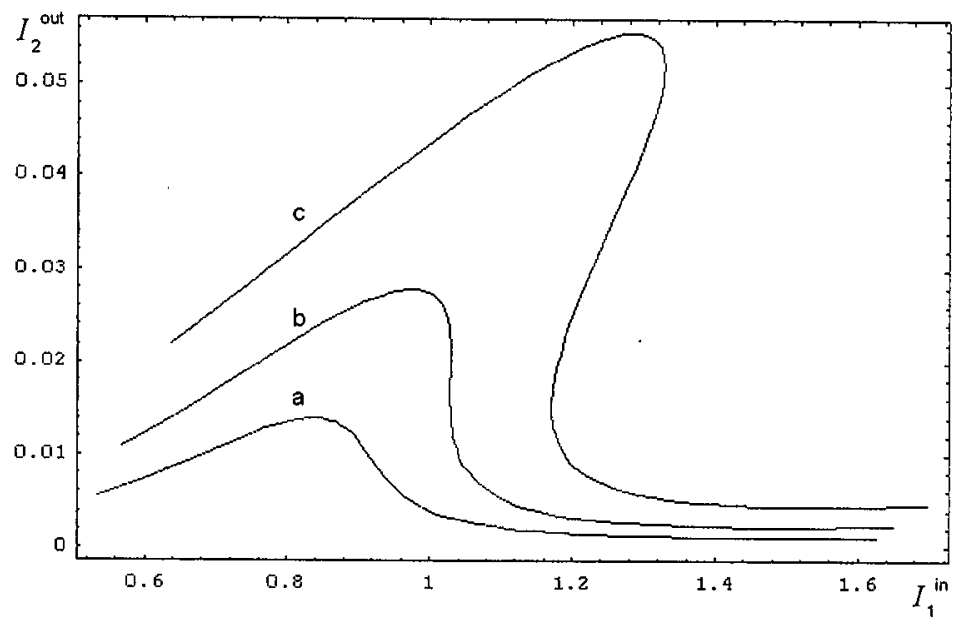

Fig. 3. Output weak-wave intensity versus strong-wave intensity for different values of input weak-wave in tensity: $a-I_{2}^{\text {in }}=0.01, b-I_{2}^{\text {in }}=0.02, c-I_{2}^{\text {in }}=0.04$ (in arbitrary units).
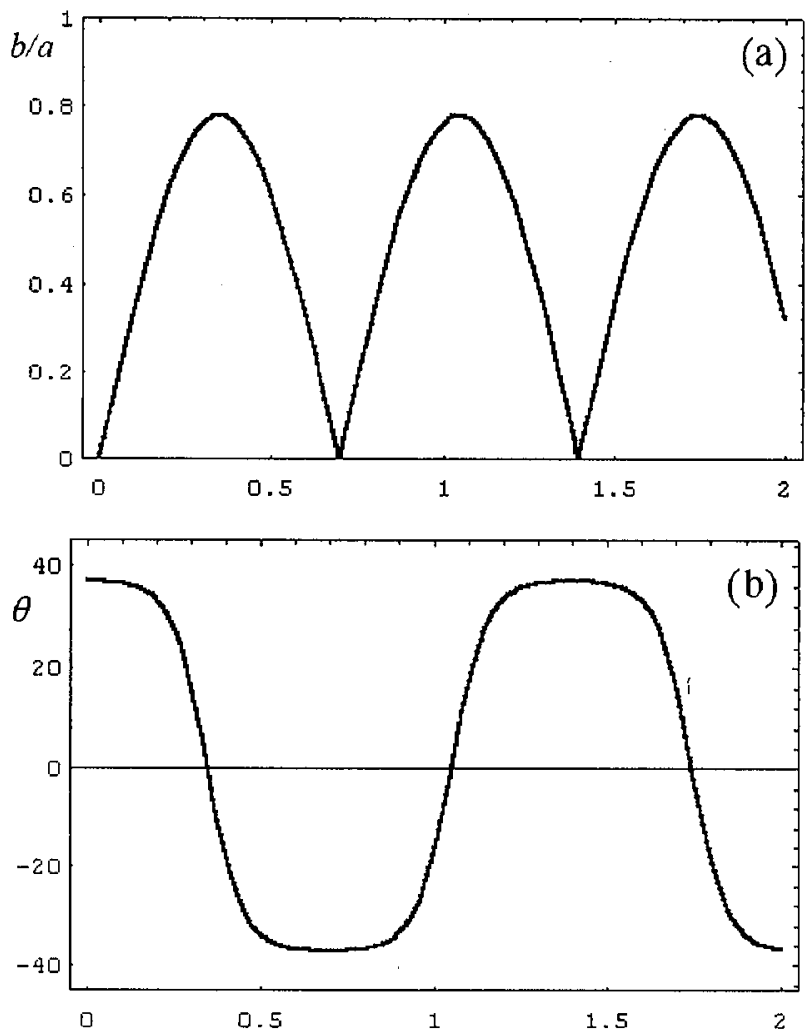

Fig. 4. (a) The ratio $b / a$ of polarisation ellipse axes of the transmitted wave as a function of the input intensity. (b) The azimuth $\theta$ (in degrees) of the polarisation ellipse axes of the transmitted wave as a function of the input intensity. 


\section{Evolution of the polarisation state}

As already mentioned, the extraordinary wave ( $\boldsymbol{E}_{y}$ component of the total electric field) of the fundamental frequency is, in our model, not influenced by the nonlinear interaction. On the other hand, the phase of the component $\boldsymbol{E}_{x}$ is intensity-dependent, as described in Secs. 2 and 3.

The polarization state is typically described in terms of Stokes parameters $\left(s_{0}, s_{1}, s_{2}, s_{3}\right)$. These are expressed by

$$
\frac{b}{a}=\tan \left(\arcsin \left|2 \frac{\sqrt{I_{x} I_{y}}}{I_{x}+I_{y}} \cos \Delta \varphi\right|\right)
$$

and the angle $\theta$ equal to

$$
\theta=\arctan \left(2 \frac{\sqrt{I_{x} I_{y}}}{I_{x}+I_{y}} \sin \Delta \varphi\right) .
$$

Here,

$$
\Delta \varphi=k z\left(n^{(\mathrm{e})}-n^{(0)}-n^{(2)} I_{x}\right)-\delta,
$$

where $\delta$ denotes the initial phase shift between $E_{x}$ and $E_{y}$ modes. Both parameters $b / a$ and $\theta$ are influenced by the nonlinear phase shift which is, as already mentioned, a function of $I_{x}$. Their dependence on $I_{x}$ for a given value of $I_{y}$ is illustrated in Figs. 4a and b.

\section{Conclusions}

The basic assumption of our calculation is that of a large wave-vector mismatch $\Delta k$. In consequence, the amplitude $A_{1}$ of the incoming wave $E_{1}$ is practically constant. However, the phase $\varphi_{1}$ of the incoming wave exhibits a dependence on the path length and a linear clependence on the intensity $I_{1}(z=0)$. The role of the second-harmonic wave $E_{2}$ with the amplitude $A_{2}$ is to bring about the path-length dependence of the phase of the incoming $E_{1}$-wave. The cascaded process is required for the appearance of the second-harmonic wave $E_{2}$, without which intensity and path-length dependence of the phase of the incoming wave $E_{1}$ cannot appear. The dependence of the nonlinear refractive index $n^{\mathrm{NL}}$ in (15) on the path length represents a new effect which is a direct consequence of the phase change $\delta \varphi_{1}(z)$ in (13). As the intensity of the second-harmonic wave with the amplitude $E_{2}$ does not appear in (13) the presence of this wave in the Fabry-Perot cavity can be neglected. The feedback process in the cavity can be described following [1, 2] by considering only the forward and backward waves of frequency $\omega$.

Bistability resulting from three-waves interaction was described in an analogous way. The wave with frequency $\omega_{3}=\omega_{1}-\omega_{2}$ takes over the role of the second-harmonic wave. This wave is indispensable for the appearance of the phase changes $\delta \varphi_{1}$ and $\delta \varphi_{2}$, however, due to its small intensity it can be neglected in this discussion of the feedback in Fabry-Perot cavity.

We thus have constructed a model mechanism of optical bistability in media without space-inversion centre, which exhibit second-order polarisation $P^{(2)}$. The number of such materials is large, however, their bistable properties have not been examined as yet in a satisfactory manner. An experimental verification 
of the conclusions of this paper for several organic materials has been recently announced [14].

\section{Acknowledgment}

This work was supported by the grant No. 8 S507 01806 of the Committee for Scientific Research.

\section{References}

[1] L.A. Lugiato, in: Progress in Optics, Vol. XXI, Ed. E. Wolf, North-Holland, Amsterdam 1984, p. 71.

[2] H.M. Gibbs, Optical Bistability, Academic Press, New York 1985.

[3] B.S. Wherrett, D.C. Hutchings, in: Nonlinear Optics in Signal Processing, Eds. R.W. Eason, A. Miller, Chapman \& Hall, London 1993, p. 145.

[4] U. Trutschel, U. Langbein, F. Lederer, H.E. Ponath, IEEE J. Quant. Electron. QE-21, 1639 (1985).

[5] B. Pura, A. Zagórski, J. Petykiewicz, Opt. Appl. 24, 229 (1994).

[6] R. Reinisch, M. Neviere, E. Popov, Opt. Lett. 20, 2472 (1995).

[7] Z.Y. Ou, Opt. Commun. 124, 430 (1996).

[8] C. Richy, K.I. Petsas, E. Giacobino, C. Fabre, L. Lugiato, J. Opt. Soc. Am. B 12, 456 (1996).

[9] A.G. White, J. Mlynek, S. Schiller, Europhys, Lett. 35, 425 (1996).

[10] R. Reinisch, E. Popov, M. Neviere, Opt. Lett. 20, 854 (1995).

[11] R. Desalvo, D.J. Hagan, M. Sheik-Bahae, G. Stegeman, E.W. van Stryland, H. Vanherzeele, Opt. Lett. 17, 28 (1992).

[12] G. Stegeman, M. Sheik-Bahae, E.W. van Stryland, G. Assanto, Opt. Lett. 18, 13 (1993).

[13] R. Schiek, J. Opt. Soc. Am. B 10, 1848 (1993).

[14] B. Pura, W. Jęda, K. Noniewicz, A. Zagórski, J. Non. Opt. Phys. Mat. 7, 441 (1998). 